\title{
Simple Analysis of XCP Equilibrium Performance
}

\author{
Peng Wang \\ Department of Electrical \& Computer Engineering \\ University of Delaware \\ Newark, DE, USA \\ Email: pwangee@udel.edu
}

\author{
David L. Mills \\ Department of Electrical \& Computer Engineering \\ University of Delaware \\ Newark, DE, USA \\ Email: mills@ece.udel.edu
}

\begin{abstract}
The recently developed eXplicit Control Protocol (XCP) is a congestion control system well suited for high Bandwidth Delay Product (BDP) networks. Low proves that the XCP equilibrium solves a constrained max-min fairness problem, and derives the explicit formulas for the link utilization and the common flow rate bottlenecked at the link by applying the derived window-based dynamic model. Furthermore, a lower bound and an upper bound of link utilization are given in Low's paper.

However, the derived window-based dynamic model is so complicated that it can not be used easily. In this paper, a simple rate-based model is proposed for analyzing XCP equilibrium performance. Most results that appeared in Low's paper can be easily reproduced by applying our simple dynamic model. Furthermore, we prove that the shuffling parameter $\gamma$ is no more than the parameter $\alpha$ to achieve high link utilization.
\end{abstract}

\section{INTRODUCTION}

The current Internet provides a connectionless, best-effort, and end-to-end packet service by using the IP protocol. The majority of the Internet traffic including HTTP, FTP, TELNET, and email traffic is carried by TCP protocol. The Van Jacobson's congestion control algorithms [1] for TCP guarantee the stability of today's Internet. However, Jacobson's algorithm also exhibits several shortcomings, such as its poor link utilization at high BDP, its unfairness at long round-trip times, and confusion by lossy links. Due to the interest in the wired network, we focus on the first two shortcomings.

Several ways are developed to improve the performance in these environments [2], [3], [4], [5], [6], [7]. Of these, the recently developed Explicit Control Protocol (XCP) [4] is a congestion control system well suited for high Bandwidth Delay Product (BDP) networks. XCP executing in the routers explicitly allocates the feedback to each packet, and the minimum feedback for each packet along the path is sent back to the sender. The per-flow state information is carried in the congestion header and the router does not maintain perflow state to avoid the scalability problem. XCP achieves high link utilization, fairness among flows with different RTTs, and reduces the packet losses. XCP is proved to be stable in the single link case when all flows have the same RTT. Simulations show that XCP is stable in more complicated

\footnotetext{
${ }^{1}$ This research is sponsored by the NSWCDD grant N00178-04-1-9001. Views and conclusions contained in this document are those of the authors and should not be interpreted as representing the official policies or endorsements of NSWCDD or the US government.
}

topologies, although the stability with a complicated topology is hard to be analytically proven.

Low [8] studies the XCP's equilibrium performance and gives some interesting results. He builds a dynamic windowbased model of XCP, proves that the XCP equilibrium solves a constrained max-min fairness problem, and derives the explicit formulas for the link utilization and the common flow rate bottlenecked at the link. Furthermore, a lower bound and an upper bound of link utilization are given in Low's paper. However, although a beautiful mathematic description of XCP is given, the derived window-based dynamic model is so complicated that it can not be used easily. Another shortcoming is that the shuffling parameter $\gamma$ and the parameter $\alpha$ can not be chosen arbitrarily which is not proven in Low's paper.

In this paper, we also focus on analysis of XCP's equilibrium performance. A simple rate-based model is proposed for analyzing XCP's equilibrium performance. Most results that appeared in Low's paper can be easily reproduced by applying our simple dynamic model. Furthermore, we prove that the shuffling parameter $\gamma$ is no more than the parameter $\alpha$ to achieve high link utilization, which is an important constraint for XCP.

The remainder of the paper is structured as follows. In the next section, XCP and Low's window-based model are introduced briefly. Then, the simple rate-based model is derived in detail. In Section III, we prove the important constraint that $\gamma \leq \alpha$ and analyze the equilibrium performance of XCP briefly. Finally, we conclude in Section IV.

$$
\text { II. Model }
$$

\section{A. XCP Congestion Control}

$\mathrm{XCP}$ is a window-based congestion control algorithm. The per-flow state information is carried in the congestion header and the router does not maintain per-flow state to avoid the scalability problem. The congestion header includes three fields: H_cwnd (sender's current cwnd), H_rtt (sender's rtt estimate) and H_feedback ( initialized to sender's demands).

XCP uses Efficiency Controller (EC) and Fairness Controller to achieve efficiency and fairness respectively. EC determines the aggregate feedback in one control interval and does not care about the per-flow feedback. The aggregate feedback $\phi$ is computed each control interval:

$$
\phi=\alpha \cdot d \cdot S-\beta \cdot Q
$$


where $\alpha$ and $\beta$ are set to 0.4 and 0.226 respectively based on the stability analysis, $d$ is the average RTT which is the control interval, $S$ is the spare bandwidth which is the difference between the flow load and the link bandwidth, and $Q$ is the persistent queue size.

The fairness controller (FC) adopts Additive-Increase Multiplicative-Decrease (AIMD) policy to achieve fairness. Additive-Increase means that the throughput of each flow increases same when $\phi>0$, and Multiplicative-Decrease means that the flow's throughput decreases proportionally to its throughput when $\phi<0$.

Moreover, XCP uses bandwidth shuffling to prevent convergence stalling when efficiency is close to optimal. It means allocation and deallocation bandwidth are executed at the same time. The shuffled traffic is:

$$
h=\max (0, \gamma \cdot y-|\phi|)
$$

where $y$ is the input traffic in an average RTT and $\gamma$ is a constant set to 0.1 . This equation assures that at least $10 \%$ of traffic is redistributed according to AIMD at every average RTT.

The feedback assigned to packet $i$ is a combination of a positive feedback $p_{i}$ and a negative feedback $n_{i}$.

$$
H_{-} \text {feedback }_{i}=p_{i}-n_{i}
$$

The per-packet positive feedback is proportional to the square of the flow's RTT, and inversely proportional to its congestion window divided by its packet size. The per-packet negative feedback is proportional to the packet size multiplied by its flow's RTT.

$$
\begin{aligned}
p_{i} & =\xi_{p} \frac{r t t_{i}^{2} \cdot s_{i}}{c w n d_{i}} \\
n_{i} & =\xi_{n} \cdot r t t_{i} \cdot s_{i}
\end{aligned}
$$

where

$$
\begin{gathered}
\xi_{p}=\frac{h+\max (\phi, 0)}{d \cdot \sum_{L} \frac{r t t_{i} \cdot s_{i}}{c w n d_{i}}} \\
\xi_{n}=\frac{h+\max (-\phi, 0)}{d \cdot \sum_{L} s_{i}}
\end{gathered}
$$

$\xi_{p}$ and $\xi_{n}$ are computed each control interval.

\section{B. Low's Window-based Dynamic Model}

Low derives a window-based dynamic model for XCP with multiple links and multiple flows. Consider a network with $L$ links shared by $N$ flows. Let $R$ be the $L \times N$ routing matrix: $R_{l i}=1$ if flow $i$ uses link $l$ and 0 otherwise. Let $L(i)$ be the set of links in the path of flow $i: L(i):=\left\{l \mid R_{l i}=1\right\}$ and $I(l)$ be the set of flows that use link $l: I(l):=\left\{i \mid R_{l i}=1\right\}$. For flow $i$, four variables are defined:

$w_{i}(t)$ : window size at time $t$, in packets.

$\tau_{i}$ : round-trip propagation (and fixed processing) delay.

$T_{i}(t)$ : round-trip time (RTT) at time $t$.

$x_{i}(t):=w_{i}(t) / T_{i}(t)$ : flow rate at time $t$.
For link $l$, four variables are defined:

$n_{l}$ : the number of flows at link $l$.

$C_{l}$ : link capacity, in packets/sec.

$b_{l}(t)$ : backlog at time $t$, in packets.

$y_{l}(t):=\sum_{i} R_{l i} x_{i}(t)$ : aggregate input rate at link $l$ at time

$t$.

The window-based dynamic model of XCP is described by the following equations:

$$
\begin{gathered}
\dot{w}_{i}(t)=\frac{w_{i}(t)}{d^{2}} \min _{l \in L(i)} F_{l i}(t) \\
\dot{b}_{l}(t)= \begin{cases}y_{l}(t)-C_{l} & \text { if } b_{l}(t)>0 \\
\max \left(y_{l}(t)-C_{l}, 0\right) & \text { if } b_{l}(t)=0\end{cases}
\end{gathered}
$$

where

$$
\begin{gathered}
F_{l i}(t)=\frac{h_{l}(t)+\phi_{l}^{+}(t)}{n_{l} x_{i}(t)}-\frac{h_{l}(t)+\phi_{l}^{-}(t)}{y_{l}(t)} \\
\phi_{l}(t)=\alpha d\left(C_{l}-y_{l}(t)\right)-\beta b_{l}(t) \\
h_{l}(t)=\max \left(\gamma d y_{l}(t)-\left|\phi_{l}(t)\right|, 0\right) \\
x_{i}(t)=\frac{w_{i}(t)}{T_{i}(t)} \\
y_{l}(t)=\sum_{i} R_{l i} x_{i}(t) \\
T_{i}(t)=\tau_{i}+\sum_{l} R_{l i} \frac{b_{l}(t)}{C_{l}}
\end{gathered}
$$

The complicated mathematic description prevents us from analyzing the equilibrium performance of XCP intuitively. In other words, the window-based model is not easily to be used.

\section{Simple Rate-based Dynamic Model}

In this section, we derive a simple rate-based dynamic model to study the equilibrium performance of XCP. We also do not consider the feedback delay because we only care about the equilibrium performance.

From the previous section of $\mathrm{XCP}$, the aggregate positive change in one control interval is $h_{l}+\max \left(\phi_{l}, 0\right)$, and the aggregate negative change in one control interval is $h_{l}+$ $\max \left(-\phi_{l}, 0\right)$ for any link $l$. It is interesting to see the values of aggregate positive and negative feedbacks of link $l$ based on the relationship of $\phi_{l}$ and $\gamma y_{l}$.

$P_{l}=h_{l}+\max \left(\phi_{l}, 0\right)=\max \left(0, \gamma y_{l}-\left|\phi_{l}\right|\right)+\max \left(\phi_{l}, 0\right)$

$$
\begin{gathered}
= \begin{cases}0 & \phi_{l} \leq-\gamma y_{l} \\
\gamma y_{l}-\left|\phi_{l}\right| & -\gamma y_{l}<\phi_{l} \leq 0 \\
\gamma y_{l} & 0<\phi_{l} \leq \gamma y_{l} \\
\phi_{l} & \gamma y_{l}<\phi_{l}\end{cases} \\
N_{l}=h_{l}+\max \left(-\phi_{l}, 0\right)=\max \left(0, \gamma y_{l}-\left|\phi_{l}\right|\right)+\max \left(-\phi_{l}, 0\right)
\end{gathered}
$$




$$
= \begin{cases}\left|\phi_{l}\right| & \phi_{l} \leq-\gamma y_{l} \\ \gamma y_{l} & -\gamma y_{l}<\phi_{l} \leq 0 \\ \gamma y_{l}-\phi_{l} & 0<\phi_{l} \leq \gamma y_{l} \\ 0 & \gamma y_{l}<\phi_{l}\end{cases}
$$

Thus, the aggregate feedback for link $l$ is

$$
P_{l}-N_{l}= \begin{cases}-\left|\phi_{l}\right| & \phi_{l} \leq-\gamma y_{l} \\ -\left|\phi_{l}\right| & -\gamma y_{l}<\phi_{l} \leq 0 \\ \phi_{l} & 0<\phi_{l} \leq \gamma y_{l} \\ \phi_{l} & \gamma y_{l}<\phi_{l}\end{cases}
$$

The simultaneous allocation and deallocation of bandwidth do not change the aggregate feedback $\phi_{l}$ at link $l$. Therefore, it shows that the efficient controller (EC) is decoupled from the fairness controller (FC).

Assume the packets from the same flow have the same $c w n d_{i}, s_{i}$, and $r t t_{i}$ during a sufficiently small time interval $d t$. The number of flow $i$ 's packets during the time interval $d t$ is

$$
\operatorname{num}_{i}=\frac{c w n d_{i} \cdot d t}{r t t_{i} \cdot s_{i}}
$$

where $c w n d_{i}$ is the flow's congestion window size, $s_{i}$ is the packet size, $r t t_{i}$ is the flow's round trip time. Thus, the flow $i$ 's aggregate positive congestion window feedback for the time interval $d t$ at link $l$ is derived from (1):

$$
p_{i a_{-} l}=p_{i_{-l} l} \cdot \text { num }_{i}=d t \cdot r t t_{i} \cdot \xi_{p l}
$$

where $p_{i_{-} l}$ is $p_{i}$ at link $l$. Furthermore, the flow $i$ 's aggregate positive throughput feedback during the interval $d t$ at link $l$ is:

$$
p_{i t h r o u g h \_l}=\frac{p_{i a_{-} l}}{r t t_{i}}=d t \cdot \xi_{p l}
$$

Similarly, the flow $i$ 's aggregate negative congestion window and throughput feedbacks can be obtained respectively for the interval $d t$ at link $l$.

$$
\begin{aligned}
& n_{i a_{-} l}=n_{i_{-} l} \cdot \operatorname{num}_{i}=d t \cdot \mathrm{cwnd}_{i} \cdot \xi_{n l} \\
& n_{i t h r o u g h \_l}=\frac{n_{i a_{-} l}}{r t t_{i}}=d t \cdot \frac{c w n d_{i}}{r t t_{i}} \cdot \xi_{n l}
\end{aligned}
$$

The change of the flow $i$ 's congestion window size $d w_{i}$ is equal to the minimum aggregate congestion window feedback during the interval $d t$ along the path.

$$
d w_{i}=\min _{l \in L(i)}\left\{d t \cdot r t t_{i} \cdot \xi_{p l}-d t \cdot \mathrm{cwnd}_{i} \cdot \xi_{n l}\right\}
$$

Assume the flow $i$ 's throughput $x_{i}$ is equal to $\frac{c w n d_{i}}{r t t_{i}}$. The change of the flow's throughput $d x_{i}$ is equal to the aggregate throughput feedback during the interval $d t$. Thus,

$$
d x_{i}=\frac{d w_{i}}{r t t_{i}}=\min _{l \in L(i)}\left\{d t \cdot\left(\xi_{p l}-x_{i} \cdot \xi_{n l}\right)\right\}
$$

Therefore, the equation can be written as:

$$
\frac{d x_{i}(t)}{d t}=\min _{l \in L(i)}\left\{\xi_{p l}-x_{i}(t) \cdot \xi_{n l}\right\}
$$

This shows that XCP implements the AIMD algorithm for each flow at each link. In the equilibrium, all flows bottlenecked in the same link have the same flow rate, and the flows bottlenecked in other links cause under-utilization problem for this link.

Since XCP is a window-based congestion control protocol, some variables are redefined to match the rate-based model. We redefine the parameter $\phi_{\text {new }}=\phi / d$ as the aggregate throughput feedback.

$$
\phi_{\text {new }}=\alpha \cdot S-\beta \cdot \frac{Q}{d}
$$

And we redefine the parameter $y_{\text {new }}=y / d$ as the aggregate flow rate. In the following sections, we still use the notation $\phi$ to represent the aggregate throughput feedback and $y$ to represent the aggregate flow rate for convenience. Then, $P$ and $N$ represent the aggregate positive and negative throughput feedbacks respectively. From (3) we know,

$$
\begin{gathered}
P_{l}=n_{l} \cdot \xi_{p} \\
N_{l}=\sum_{i \in I(l)} x_{i}(t) \cdot \xi_{n}=y_{l} \cdot \xi_{n}
\end{gathered}
$$

where $y_{l}$ is the aggregate flow rate and $n_{l}$ is the number of flows at link $l$.

In summary, the simple rate-based dynamic model is described by the following equations.

$$
\frac{d x_{i}(t)}{d t}=\min _{l \in L(i)}\left\{\xi_{p l}-x_{i}(t) \cdot \xi_{n l}\right\}
$$

where

$$
\begin{gathered}
\xi_{p l}= \begin{cases}0 & \phi_{l} \leq-\gamma y_{l} \\
\frac{\gamma y_{l}-\left|\phi_{l}\right|}{n_{l}} & -\gamma y_{l}<\phi_{l} \leq 0 \\
\frac{\gamma y_{l}}{n_{l}} & 0<\phi_{l} \leq \gamma y_{l} \\
\frac{\phi_{l}}{n_{l}} & \gamma y_{l}<\phi_{l}\end{cases} \\
\xi_{n l}= \begin{cases}\frac{\left|\phi_{l}\right|}{y_{l}} & \phi_{l} \leq-\gamma y_{l} \\
r & -\gamma y_{l}<\phi_{l} \leq 0 \\
\frac{\gamma y_{l}-\phi_{l}}{y_{l}} & 0<\phi_{l} \leq \gamma y_{l} \\
0 & \gamma y_{l}<\phi_{l}\end{cases}
\end{gathered}
$$

Note: The flow $i$ 's rate is determined by the bottleneck link. This means that we only need to consider the bottleneck link for flow $i$ to determine the flow $i$ 's rate.

\section{Simple analysis of link utilization}

In this section, we analyze the XCP's equilibrium performance briefly. Let us consider a link $l$. The flows traversing through link $l$ are classified into three sets. One set denoted by $\varphi$ contains the flows which are bottlenecked at the downstream links. Another set denoted by $\chi$ contains the flows which are bottlenecked at the upstream links. The last set denoted by $\psi$ contains the flows which are bottlenecked at link $l$ itself.

Furthermore, all links can be classified into five typical classes based on the flows traversing through. 
Class 1: $\psi=\emptyset ; \chi$ do not care; $\varphi$ do not care

All flows are bottlenecked elsewhere. Since the router can not allocate any spare bandwidth to flows, it is not meaningful to discuss the link utilization in this case. The link utilization can be arbitrarily small as the link bandwidth is arbitrarily large when all flows are controlled by other routers.

Class $2: \psi \neq \emptyset ; \chi=\emptyset ; \varphi=\emptyset$

In the equilibrium, all flows in set $\psi$ have the flow rate:

$$
x_{i}=\frac{\xi_{p l}}{\xi_{n l}}
$$

From the equation (7),

$$
y_{l}=N_{l} \cdot x_{i}=\frac{\gamma y_{l}^{2}}{\gamma y_{l}-\alpha \cdot\left(C_{l}-y_{l}\right)}
$$

Thus, the aggregate flow rate $y_{l}$ is equal to the link capacity $C_{l}$, and the link is fully utilized. Furthermore, the per-flow rate is $x_{i}=C_{l} / N_{l}$ and the max-min fairness is achieved.

Class $3: \psi \neq \emptyset ; \chi=\emptyset ; \varphi \neq \emptyset$

All flows are bottlenecked in the downstream links and the current link. In the equilibrium the feedback for flows in set $\psi$ is

$$
\xi_{p l}-x_{i} \cdot \xi_{n l}=0 \quad i \in \psi
$$

It is obvious that the flows in set $\psi$ have a higher flow rate than the flows in set $\varphi$. Otherwise, the flows will be bottlenecked in the current link. Thus, the feedback for flows in set $\varphi$ is

$$
\xi_{p l}-x_{j} \cdot \xi_{n l}>0 \quad j \in \varphi
$$

The feedback for flows $j \in \varphi$ is eliminated at the downstream links, and the under-utilization problem of XCP appears. The detailed discussion is given in the following section.

Class $4: \psi \neq \emptyset ; \chi \neq \emptyset ; \varphi=\emptyset$

All flows are bottlenecked in the upstream links and the current link. In the equilibrium, the feedback for flows in set $\chi$ is 0 , but the current link still allocates feedbacks to flows in set $\chi$. In fact, XCP treats the flows in set $\chi$ the same as the flows in set $\psi$ due to the AIMD. It causes the same under-utilization problem as that of Class 3.

Since the flows in set $\chi$ can be detected at the current link in the equilibrium, Katabi [4] tries to alleviate the negative effects caused by the flows in set $\chi$ with the pseudo code in Fig. 1.

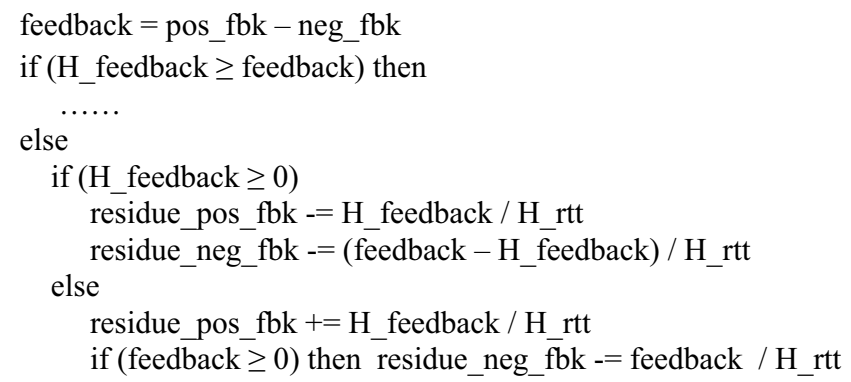

Fig. 1. Compensation pseudo code of XCP
The compensation policy affects the equilibrium, but it only increases the link utilization by several percentage points. The deterministic factor is still the derivative equation which is the description of XCP. The detailed analysis of the above compensation policy is a topic for further research.

Class $5: \psi \neq \emptyset ; \chi \neq \emptyset ; \varphi \neq \emptyset$

In the equilibrium, the link utilization should be not worse than that of Class 3 and not better than that of Class 4 if the compensation policy is adopted. Otherwise, it is same as Class 3.

In summary, we call the network analysis Downstream case if the compensation policy is not adopted. Otherwise, we call it Upstream case. In Low's analysis, the compensation policy is not considered. The flows bottlenecked at the upstream links and downstream links are treated equally according to AIMD. Therefore, Low's analysis is same as our analysis of Downstream case. In the following section, we focus on studying Downstream case and give some interesting analysis.

\section{Link Utilization OF Downstream CASE}

If all flows are bottlenecked at other links and link $l$ can not allocate any spare bandwidth to any flows, it is not meaningful to analyze the link utilization in this case since the link utilization can be arbitrarily small as the link capacity approaches to infinite. Thus, we have an important assumption for all of our analysis.

Assumption: Assume the set $\psi$ of link $l$ is not empty in all analysis.

\section{A. Simple and Important Results}

In this section, we derive some simple and important results of XCP.

Lemma 1: In the equilibrium, $0 \leq \phi_{l} \leq \gamma y_{l}$.

Proof: In the equilibrium, assume $\gamma y_{l}<\phi_{l}$, the aggregate positive feedback $P_{l}=\phi_{l}$ and the aggregate negative feedback $N_{l}=0$. Thus, the negative feedback for all flows is 0 . However, the flows in set $\psi$ satisfy

$$
\frac{d x_{i}}{d t}=\xi_{p l}>0
$$

It means that these flows are not in the equilibrium. By contradiction, we know that $\phi_{l} \leq \gamma y_{l}$.

If $\phi_{l}<0$, the feedback filled in the packet header is negative no matter whether the flow is bottlenecked in the upstream link or this link. Moreover, downstream links can replace this negative feedback only if they have more negative feedback. Thus, the final feedback is always negative and the flow rate has to be decreased. It means that the flows are not in the equilibrium. We conclude that $0 \leq \phi_{l} \leq \gamma y_{l}$.

Lemma 2: In the equilibrium, the queue $Q_{l}$ is empty and the aggregate throughput feedback is $\phi_{l}=\alpha \cdot\left(C_{l}-y_{l}\right)$.

Proof: In the equilibrium, the aggregate flow rate is no more than the link capacity. Otherwise, the flows can not achieve the equilibrium.

If the aggregate flow rate $y_{l}$ is less than the link capacity $C_{l}$, it is obviously that the queue $Q_{l}$ is empty. If the aggregate 
flow rate $y_{l}$ is equal to the link capacity $C_{l}$, we assume the queue $Q_{l}$ is not empty in the equilibrium. Then

$$
\phi_{l}=\alpha \cdot\left(C_{l}-y_{l}\right)-\beta \cdot \frac{Q_{l}}{d}<0
$$

Thus, the flows are not in the equilibrium from Lemma 1. We conclude that $Q_{l}$ is empty. Furthermore, the aggregate throughput feedback is $\phi_{l}=\alpha \cdot\left(C_{l}-y_{l}\right)$.

Theorem 1: In the equilibrium, if all links have $\gamma \rightarrow 0$, the network approaches the max-min fairness.

Proof: We only need to consider the bottlenecks in the network. From lemma 1 and 2, we know that set $\psi$ of link $l$ is not empty and link $l$ has the aggregate feedback $\phi_{l}=\alpha\left(C_{l}-y_{l}\right) \rightarrow 0$ as $\gamma \rightarrow 0$. Then, link $l$ is fully utilized and all flows bottlenecked at link $l$ have the same flow rate $x_{l}=\xi_{p l} / \xi_{n l}$ which is also the maximum allowable flow rate at link $l$. Moreover, $x_{l}$ can not be increased without decreasing the rates of flows which are not bottlenecked at link $l$ because $x_{l}$ is the maximum flow rate and link $l$ is fully utilized. Similarly, every bottleneck link works like link $l$. Thus, the network approaches the max-min fairness.

Note: If we substitute $r=0$ to Low's derived common flow rate formula, the max-min fairness can also be proven.

\section{B. Reproduce Low's Results}

Low derives a dynamic window-based model for XCP and analyzes the link utilization and the fairness. It gives many encouraging results. To compare with Low's results, we use the same notations in our following analysis.

$N_{l}$ : the number of flows at link $l$

$N_{l 0}$ : the number of flows bottlenecked at other routers

$y_{l 0}$ : the aggregate flow rate bottlenecked at other routers

$\rho_{l}:=N_{l 0} / N_{l}$

$\sigma_{l}:=y_{l 0} / C_{l}$

Since the compensation policy is not considered here, the flows bottlenecked at the upstream links and downstream links are treated equally by XCP. We can use the above notations without differentiating the upstream and downstream bottlenecked flows in the theoretical analysis.

Let us see Low's work first. The formula given by Low to calculate the equilibrium flow rate bottlenecked at link $l$ is based on the following equation.

$$
\frac{y_{l}-y_{l 0}}{N_{l}-N_{l 0}}=r^{l}=\frac{\gamma \cdot y_{l}^{2}}{N_{l} \cdot\left(\gamma y_{l}-\alpha\left(C-y_{l}\right)\right)}
$$

This is a key equation in Low's paper. Most of his results are obtained from this equation directly or indirectly, such as the aggregate flow rate $y_{l}$, the equilibrium flow rate $x_{r}$ at link $l$, and the upper and lower bounds of link utilization.

In our model, the same formula can be easily derived for Downstream case. The equilibrium flow rate bottlenecked at link $l$ is

$$
x_{r}=\frac{\xi_{p l}}{\xi_{n l}}
$$

where

$$
\xi_{p l}=\frac{\gamma \cdot y_{l}}{N_{l}}
$$

$$
\xi_{n l}=\frac{\gamma \cdot y_{l}-\alpha\left(C_{l}-y_{l}\right)}{y_{l}}
$$

Thus,

$$
\frac{y_{l}-y_{l 0}}{N_{l}-N_{l 0}}=x_{r}=\frac{\gamma \cdot y_{l}^{2}}{N_{l} \cdot\left(\gamma y_{l}-\alpha\left(C_{l}-y_{l}\right)\right)}
$$

which is same as Low's formula. Therefore, most of Low's results can be reproduced by applying our model.

C. $\gamma$ and $\alpha$

1) The relationship of $\gamma$ and $\alpha$ : Low does not give a constraint on the relationship of $\gamma$ and $\alpha$, but his simulations show that the link utilization is very low if $\gamma / \alpha$ approaches to a large value. In fact, the shuffling parameter $\gamma$ and the parameter $\alpha$ can not be chosen arbitrarily. We show that the shuffling parameter $\gamma$ is no more than the parameter $\alpha$ to achieve high link utilization in this section.

Let us see the relationship between $x_{r} N_{l}$ and the link capacity $C_{l}$ first where $x_{r}$ represents the common flow rate bottlenecked at link $l$. If all flows belong to set $\psi$, we can get $x_{r} N_{l}=C_{l}$ which is easy to check. On the other hand, if some flows belong to set $\chi$ and set $\varphi$, the common flow rate $x_{r}$ is obviously greater than the flow rate $x_{j}, j \in \chi, \varphi$. If $x_{r} N_{l} \leq C_{l}$, the under-utilization problem is obviously worse. It is necessary to guarantee $x_{r} N_{l} \geq C_{l}$.

Theorem 2: To guarantee $x_{r} N_{l} \geq C_{l}$ where $x_{r}$ represents the common flow rate bottlenecked at the current link $l$, the shuffling parameter $\gamma$ and the parameter $\alpha$ must satisfy $\gamma \leq$ $\alpha \frac{C_{l}}{y_{l}}$. Furthermore, we choose $\gamma \leq \alpha$ to guarantee that $\gamma \leq$ $\alpha \frac{C_{l}}{y_{l}}$.

Proof: In the equilibrium, the flows in set $\psi$ have a common flow rate $x_{r}$, and the feedback of these flows is 0 . Thus,

$$
\begin{gathered}
\xi_{n l}=\frac{\gamma \cdot y_{l}-\alpha\left(C_{l}-y_{l}\right)}{y_{l}} \\
\xi_{p l}=x_{r} \cdot \xi_{n l}=x_{r} \cdot \frac{\gamma \cdot y_{l}-\alpha\left(C_{l}-y_{l}\right)}{y_{l}}
\end{gathered}
$$

The bandwidth wasted by the flows in set $\varphi$ or set $\chi$ is equal to the allocated bandwidth $\phi_{l}$ in the equilibrium. Then,

$$
\begin{aligned}
& \alpha\left(C_{l}-y_{l}\right)=\sum_{j \in \varphi, \chi}\left(\xi_{p l}-x_{j} \cdot \xi_{n l}\right) \\
= & \frac{\gamma \cdot y_{l}-\alpha\left(C_{l}-y_{l}\right)}{y_{l}} \cdot \sum_{j \in \varphi, \chi}\left(x_{r}-x_{j}\right) \\
= & \frac{\alpha y_{l}+\gamma y_{l}-\alpha C_{l}}{y_{l}} \cdot\left(x_{r} N_{l 0}-y_{l 0}\right)
\end{aligned}
$$

Finally,

$$
x_{r} N_{l 0}-y_{l 0}=\frac{\alpha}{\alpha+\frac{\gamma y_{l}-\alpha C_{l}}{y_{l}}} \cdot\left(C_{l}-y_{l}\right)
$$

If $\gamma \leq \alpha \frac{C_{l}}{y_{l}}$, then

$$
x_{r} N_{l 0}-y_{l 0}+y_{l} \geq C_{l}
$$


Therefore,

$$
x_{r} N_{l} \geq C_{l}
$$

Furthermore, since we know that $y_{l} \leq C_{l}$, we choose $\gamma \leq \alpha$ to guarantee that $\gamma \leq \alpha \frac{C_{l}}{y_{l}}$.

2) Choosing values of $\gamma$ and $\alpha$ : Low gives the lower and upper bounds of link l's utilization:

$$
\frac{\alpha}{\gamma \rho_{l}+\alpha} \leq \frac{y_{l}}{C_{l}} \leq 1-\frac{\gamma \sigma_{l}\left(\rho_{l}-\sigma_{l}\right)}{\gamma \rho_{l}+\alpha}
$$

From the control theorem, we know that the parameters $\gamma$ and $\alpha$ are controllable, while the parameters $\rho_{l}$ and $\sigma_{l}$ are observable instead of controllable. Due to the diversity of $\rho_{l}$ and $\sigma_{l}$, the relaxed bounds are derived only based on the controllable variables $\gamma$ and $\alpha$. Since the set $\psi$ is not empty, $\rho_{l}$ belongs to the interval $[0,1)$. The relaxed bounds are given as

$$
\frac{\alpha}{\alpha+\gamma}<\frac{y_{l}}{C_{l}} \leq 1
$$

Let us define $u$ as the desired link utilization, and $u$ belongs to the interval $(0.5,1]$ because $\gamma \leq \alpha$. Therefore, the chosen lower bound must be no less than $u$ to guarantee the desired link utilization $u$. Thus, we get a relationship of $\gamma$ and $\alpha$.

$$
\frac{\gamma}{\alpha} \leq \frac{(1-u)}{u}
$$

Fig. 2 shows the feasible values of $\gamma / \alpha$ for the given desired link utilization $u$.

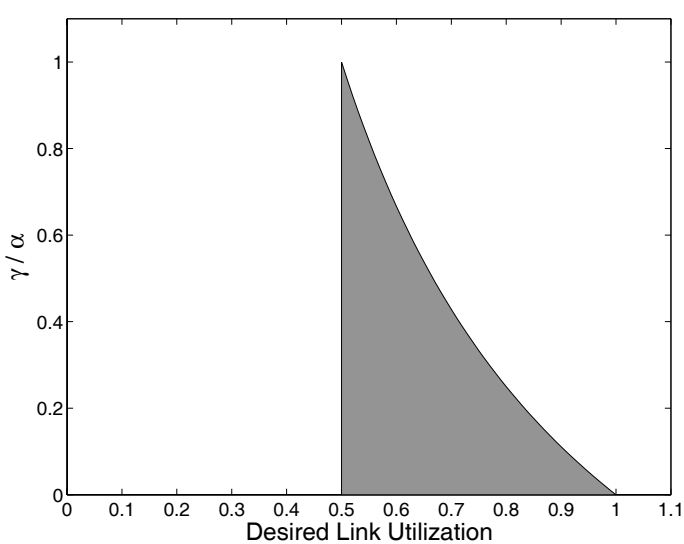

Fig. 2. Feasible values of $\frac{\alpha}{\gamma}$ for Desired Link Utilization

\section{Conclusion}

In this paper, we focus on analysis of XCP's equilibrium performance. A simple rate-based model is proposed for analyzing the XCP's equilibrium performance. Most results that appeared in Low's paper can be easily reproduced by applying our simple dynamic model. Furthermore, we prove that the shuffling parameter $\gamma$ is no more than the parameter $\alpha$ to achieve high link utilization, which is an important constraint for XCP.
Further research includes analyzing the effect of the compensation policy. The compensation policy increases the link utilization by several percentage points, and we try to find the rules behind it. Another topic is that the link utilization may have more stringent bounds provided with the new constraint $\gamma \leq \alpha$

\section{REFERENCES}

[1] V. Jacobson, "Congestion avoidance and control," in Proc. ACM SIGCOMM '88, Stanford, CA, USA, 1988, pp. 314-329.

[2] A. Falk, T. Faber, J. Bannister, A. Chien, R. Grossman, and J. Leigh, "Transport protocols for high performance," Communications of ACM, vol. 46, no. 11, pp. 43-49, 2003.

[3] F. Kelly, A. Maulloo, and D. Tan, "Rate control in communication networks: shadow prices, proportional fairness and stability," Journal of the Operational Research Society, vol. 49, no. 3, pp. 237-252, 1998.

[4] D. Katabi, M. Handley, and C. Rohrs, "Congestion control for high bandwidth-delay product networks," in Proc. ACM SIGCOMM'02, Pittsburgh, PA, USA, 2002, pp. 89-102.

[5] T. Kelly, "Scalable tcp: Improving performance in highspeed wide area networks," ACM SIGCOMM Computer Communication Review, vol. 33, no. 2, pp. 83-91, 2003.

[6] C. Jin, D. Wei, and S. Low, "Fast tcp: Motivation, architecture, algorithms, and performance," in Proc. IEEE Infocom, Hongkong, 2004.

[7] S. Floyd, "HighSpeed TCP for large congestion windows," RFC 3649 , 2003.

[8] S. H. Low, L. L. H. Andrew, and B. P. Wydrowski, "Understanding XCP: Equilibrium and fairness," in Proc. IEEE Infocom, Miami, FL, USA, 2005. 